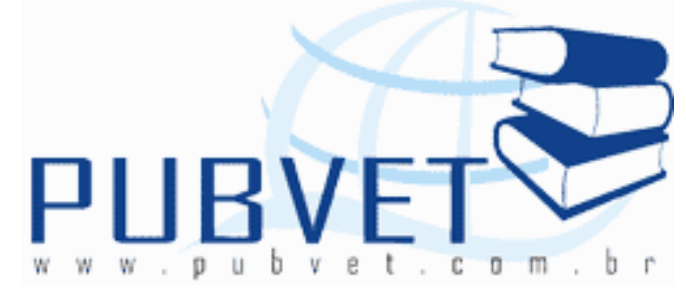

PUBVET, Publicações em Medicina Veterinária e Zootecnia.

Disponível em: <https://doi.org/10.31533/pubvet.v02n11a428>.

\title{
Aplicação da técnica da taxa de depuração renal de creatinina para avaliação do "status" do cálcio em eqüinos ${ }^{1}$
}

Paulo Reis de Carvalho², Raul Dantas d'Arce ${ }^{3}$, Raul Machado Neto ${ }^{3}$, Airton Manzano 4

${ }^{1}$ Apoio financeiro da EMBRAPA - UEPAE, São Carlos-SP.

2 Parte da Dissertação de mestrado do primeiro autor.

2 Méd. Vet., Dr., Pesquisador, APTA Regional - Secretaria Agricultura e Abastecimento, Bauru-SP.

3 Professor da Escola Superior de Agricultura Luiz de Queiroz - ESALQ/USP, Piracicaba - SP.

${ }^{4}$ Engo Agro., Dr., Pesquisador, EMBRAPA-UEPAE, São Carlos-SP.

Resumo - Este experimento foi realizado com objetivo de avaliar a eficiência da determinação da taxa do "clearance" de creatinina para o cálcio (\% DRCr- Ca) como ferramenta de diagnóstico de osteopatias em eqüinos recebendo dietas com teores variáveis ou desequilibradas em Ca na ração. Foram utilizadas 20 potras árabes e mestiça árabe com idade média de 19,9 meses, em delineamento inteiramente casualizado, com cinco potras por unidade experimental, submetidas a quatro tratamentos para coletas diárias 
de sangue e urina nos tempos de 0, 48, 72, 96 e 120 horas após período de adaptação de 15 dias. As rações dos tratamentos com diferentes níveis de $\mathrm{Ca} /$ dia na dieta foram compostas de: A- Ca normal e fósforo $(P)$ normal - $24 \mathrm{~g}$ de Ca e $18 \mathrm{~g}$ de P/dia; B- Ca baixo e P alto - 4,42 g de Ca e 30,10 g de P/dia; C - Ca baixo e P baixo - 15,6 g de Ca e 9,6 g de P/dia; e D - Ca alto e P baixo 29,04 $\mathrm{g}$ de Ca e 14,04 g de P/dia na dieta. Os animais receberam $6 \mathrm{~kg}$ de MS/dia em duas refeições. O \% DRCr- Ca e respectivos níveis de significância para as médias nos tratamentos e nos tempos variaram de: $\mathrm{A}: 3,52 \%(0 \mathrm{~h})$ a $10,15 \%$ (96 h), P>0,05; B: 5,10\% (0 h) a 0,79\% (72 h), P<0,01; C: 3,50\% (0 h) a $1,43 \%(72 \mathrm{~h}), \mathrm{P}<0,05$; e $\mathrm{D}: 6,89 \%(0 \mathrm{~h})$ a $11,42 \%(120 \mathrm{~h}), \mathrm{P}>0,05$. As dietas com Ca deficiente nos grupos $\mathrm{B}(48 \mathrm{~h}-1,22 \%, \mathrm{P}<0,01)$ e $\mathrm{C}(72 \mathrm{~h}-$ $1,43 \%, \mathrm{P}<0,01)$ mostraram decréscimos significativos do \% $\mathrm{DRCr}-\mathrm{Ca}$ entre tempos e tratamentos, respectivamente, e decréscimos da excreção de $\mathrm{Ca}$ urinário, permitindo concluir que é possível à detecção precoce da deficiência de Ca no animal com \%DRCr-Ca menores que 3,50\%.

Palavras-chave: taxa clearance, depuração renal, osteodistrofia, urina, potra árabe

\section{Application of technique of the renal purification of creatinine ratio for evaluation of the calcium status in equine}

Abstract - This experiment was carried out to evaluate the efficiency of creatinine clearance ratios for the calcium (\% DRCr-Ca) as tool of diagnosis in the prevention of the osteopathology in equines receiving diets unbalanced in Ca. Twenty fillies averaging 19,9 months of age Arabian and crossbred Arabian fillies were used, in completely randomized design, with five fillies for experimental unit, submitted the four treatments for daily collections of blood and urine in the times of $0,48,72,96$ and 120 hours after period of adaptation of fifteen days. The diets of treatments with different levels of $\mathrm{Ca} /$ day in the diet was composed of: The normal $\mathrm{Ca}$ and normal $\mathrm{P}-24 \mathrm{~g}$ of $\mathrm{Ca}$ and $18 \mathrm{~g}$ of P/day; B - Ca low and high P - 4,42 g of Ca and 30,10 g of P/day; C - 
low $\mathrm{Ca}$ and low P - 15,6 g of Ca and 9,6 g of P/day; and D - high Ca and low P - 29,04 $\mathrm{g}$ of $\mathrm{Ca}$ and $14,04 \mathrm{~g}$ of $\mathrm{P} /$ day in the diet. The animals received six $\mathrm{kg}$ of MS/day in two meals. The \% DRCr- Ca and respective levels of significant to averages in the treatments and the times had varied: $3.52 \%(0 \mathrm{~h}) 10.15 \%$ (96 h), and P>0.05; B: 5.10\% (0 h) 0.79\% (72 h), and P<0.01; C: 3.50\% (0 h) $1.43 \%(72 \mathrm{~h})$, and $\mathrm{P}<0.05$; and $\mathrm{D}:$ : 6.89\% ( $0 \mathrm{~h}) 11.42 \%(120 \mathrm{~h})$, and $\mathrm{P}>0.05$. The diets with deficient $\mathrm{Ca}$ in groups $\mathrm{B}(48 \mathrm{~h}-1,22 \%, \mathrm{P}<0.01)$ and $\mathrm{C}(72 \mathrm{~h}-$ $1.43 \%, \mathrm{P}<0.01)$ showed significant decreases of \% DRCr- Ca between times and treatments, respectively, and decreases of the excretion of urinary Ca, allowing it is possible to conclude the precocious detection of the $\mathrm{Ca}$ in animal deficiency with \%DRCr-Ca lower than $3.50 \%$.

Key Words: clearance ratio, renal depuration, osteodystrophy, urine, arabian filly

\section{Introdução}

O esqueleto em mamíferos apresenta duas e às vezes incompatíveis funções, a estrutural e a metabólica. Metabolicamente, o esqueleto funciona principalmente como reservatório de íons de vital importância, entre os quais o cálcio ( $\mathrm{Ca}$ ). No osso e cartilagem, hormônios atuam de maneira coordenada a manter o balanço entre estas duas funções (Raisz \& Kream, 1981). Os tecidos do esqueleto detém entre 80 e $85 \%$ de toda matéria mineral do organismo animal, sendo mais de $99 \%$ do Ca corporal encontrado nos ossos e dentes. Segundo Wasserman (1984), o mineral ósseo de estrutura cristalina da série apatita é formado de hidroxiapatita de formula estrutural $\mathrm{Ca}_{10}\left(\mathrm{PO}_{4}\right)_{6}(\mathrm{OH})_{2}$ e sais de Ca do tipo fosfato tricalcio e carbonato de cálcio. De acordo com Krook (1968), a renovação constante de tecido ósseo é a base para classificação da morfologia e patogenia de doença metabólica óssea caracterizada por osteopenia.

O rim é o principal regulador da homeostasia do Ca no fluído extracelular em eqüinos e segundo Nordin \& Peacock (1969) para equivalente estágio de 
maturidade e ingestão de cálcio por bovinos e ovinos, cavalos absorvem mais $\mathrm{Ca}$ e excretam maior proporção de $\mathrm{Ca}$ absorvido na urina. A proporção de $\mathrm{Ca}$ absorvido e excretado pelos rins do eqüino aumenta numa relação direta a absorção. Os rins de cavalos em crescimento excretam média aproximada de $30 \%$ do Ca absorvido e do cavalo de 18 a 24 meses, entre 40 a 50\% do Ca absorvido (Schryver et al, 1974). A grande quantia de carbonato de $\mathrm{Ca}$ encontrada na urina eqüina segundo Brobst et al. (1978) confirma a importância do rim como meio de excreção de Ca na manutenção da homeostasia do Ca plasmático.

A absorção de Ca da dieta pode variar de 45 a $70 \%$ e a perda fecal endógena tem apresentado média de 20 mg/kg PV/dia (Schryver et al., 1970a; Elfers et al., 1986), a excreção endógena renal de $5 \mathrm{mg}$ a $6 \mathrm{mg} \mathrm{Ca} / \mathrm{kg} \mathrm{PV/dia}$ em cavalos ingerindo Ca próximo dos requerimentos de mantença (Schryver et al., 1970a, 1974; Blaney et al., 1981) e $18 \mathrm{mg}$ a $20 \mathrm{mg} \mathrm{Ca} / \mathrm{kg} \mathrm{PV} / \mathrm{dia}$ em cavalos com dieta a base de feno de alfafa $(1,52 \% \mathrm{Ca})$ e cereais (Cymbaluk \& Christensen, 1986).

Dificuldade encontrada na obtenção de coleção total de urina no tempo de 24 horas, especialmente em cavalo, segundo Handa (1967) e Lane (1983), pode ser resolvida adotando-se técnica simplificada na rotina clínica baseada em que a depuração de uma substancia, $X$, que requer somente concentração de $\mathrm{X}$ e creatinina $(\mathrm{Cr}$ ) em coleta simultânea de amostra de urina e soro sangüíneo para determinação do clearance de creatinina para o íon X (\% Cr$X)$. Experimentalmente, Morris et al. (1984) observou que a excreção de $\mathrm{Cr}$ permanece praticamente constante num período de 24 horas em condições de homogeneidade de práticas de manejo incluindo principalmente alimentação e atividade física. Knudsen (1959) demonstrou em cavalos que o clearance de creatinina endógena tem valor relativo ao redor de $90 \%$ em relação ao clearance de inulina e é usado nas determinações de $\mathrm{Cr}$ para avaliação da filtração glomerular renal no cavalo (Gans \& Mercer, 1984).

O objetivo desta pesquisa foi determinar o \% DRCr- Ca em amostra de urina e soro sangüíneo colhidos simultaneamente de eqüinos sadios 
submetidos às dietas com diferentes porcentuais de $\mathrm{Ca}$ nas rações experimentais com o propósito de avaliar o efeito dos tratamentos e do tempo sobre o status nutricional e metabólico do Ca. Averiguar a eficácia da técnica $\%$ DRCr- em detectar precocemente as variações do perfil bioquímico do íon Ca e avaliar a função renal dos eqüinos submetidos às dietas com diferentes teores de Ca.

\section{Material e Métodos}

A fase experimental do presente projeto de pesquisa foi desenvolvida na Unidade de Pesquisa de Âmbito Estadual da EMBRAPA de São Carlos-SP, clima tipo $\mathrm{CW}$, subtropical chuvoso, inverno seco e precipitação anual média de $1502 \mathrm{~mm}$.

Foram utilizadas 20 potras, sendo 10 potras puras da raça Árabe e 10 mestiças Árabe, com idade média de 19,9 meses. O peso e altura médios das potras e o desvio padrão no início do experimento foram, respectivamente, de $277,2 \mathrm{~kg} \pm 37,76$ e $1,37 \mathrm{~m} \pm 0,05$. O peso e altura médios das potras e 0 desvio padrão no final do experimento foram, respectivamente, de $284,8 \mathrm{~kg} \pm$ 33,95 e $1,40 \mathrm{~m} \pm 0,04$.

Os animais foram distribuídos em baias individuais de alvenaria com área de $11,97 \mathrm{~m}^{2}(4,20 \mathrm{~m} \times 2,85 \mathrm{~m})$, piso de cimento, providas de cocho de cimento onde recebiam a ração de concentrado e volumoso nos tratamentos especificados. Durante as fases de adaptação e experimental não foram usados qualquer tipo de cama sobre o piso.

Os tratamentos, em número de quatro, foram aplicados a grupos de cinco potras, distribuídos a seguir: A- cálcio normal e fósforo normal - $24 \mathrm{~g}$ de Ca e $18 \mathrm{~g}$ de $\mathrm{P}$ por dia na dieta para atender as exigências dos animais desta faixa etária; B- cálcio baixo e fósforo alto - 4,42 g de Ca e 30,10 g de P por dia na dieta; C - cálcio baixo e fósforo baixo - 15,6 g de Ca e 9,6 g de P por dia na dieta; D - cálcio alto e fósforo baixo - 29,04 g de Ca e 14,04 g de P por dia na dieta. Os animais receberam $6 \mathrm{~kg}$ de MS/dia, sendo a primeira refeição as $10 \mathrm{~h}$ 
e a segunda as $16 \mathrm{~h}$ (Tabela 1 ). A composição química bromatológica dos ingredientes das dietas experimentais foram determinadas segundo a metodologia descrita pelo AOAC (1970). A composição mineral em Ca e P das dietas experimentais foram determinadas espectrofotometria de absorção atômica da marca $\operatorname{Varian}^{\circledR}$.

\section{Análises bioquímicas}

O sangue total para obtenção do soro foi colhido por punção da jugular em tubo de vidro tipo vacutainer sem anticoagulante. A urina foi colhida diretamente da bexiga com auxílio de sonda metálica de inox esterelizada e precedida de cuidadosa assepsia do local. Após 15 dias de adaptação, as amostras de urina e soro foram obtidas diariamente com colheita simultânea no horário entre 12 h e $14 \mathrm{~h}$ nos tempos 0 (inicial), 48, 72, 96 e $120 \mathrm{~h}$ do início da fase experimental. A urinálise foi realizada após centrifugação de alíquota de amostra de urina para estudo de sedimentoscopia de cristais, células de descamação e flora presentes no aparelho urinário.

O Ca e Creatinina ( $\mathrm{Cr}$ ) no soro e urina, para determinação do clearance percentual de Ca (\% DRCr-Ca), foram dosados pelo método kit Labtest $^{\circledR}$ Sistemas Diagnósticos Ltda., por reação colorimétrica com leitura em espectrofotômetro de absorção molecular modelo micronal 382-B.

O ácido oxálico no feno de Coast cross foi determinado pela técnica de cromatografia em camada delgada utilizando como controle na corrida cromatográfica solução padrão de ácido oxálico de concentração conhecida para teste de identificação e quantificação da solução da amostra pesquisada.

$\mathrm{Na}$ análise estatística dos dados, cada tratamento foi aplicado a cada unidade inteiramente ao acaso, a cinco animais, de acordo com o modelo matemático: $Y i j=m+t i+e i j$. Os resultados das determinações do \%DRCr$\mathrm{Ca}$, $\mathrm{Ca}$ e $\mathrm{Cr}$ no soro e urina foram analisados nos tempos de 0,48, 72, 96 e $120 \mathrm{~h}$ para cada unidade experimental. Para comparar as médias das variáveis estudadas entre tratamentos foi realizada a análise conjunta dos tempos dentro do tratamento. Os dados foram processados mediante o uso do 
Carvalho, P.R. d'Arce, R.D., Machado Neto, R., et al. Aplicação da técnica da taxa de depuração renal de creatinina para avaliação do "status" do cálcio em eqüinos. PUBVET, Londrina, V. 2, N. 44, Art\#428, Nov1, 2008.

software "Statistical Analysis System" (SAS, 1994). O efeito do tempo foi estudado através da regressão polinomial, enquanto que as médias dos tratamentos foram comparadas pelo teste de Tukey ao nível de significância indicado.

Tabela 1 - Composição das rações experimentais

\begin{tabular}{l|c|c|c|c}
\hline \multirow{2}{*}{ Ingredientes (\%) } & \multicolumn{5}{|c}{ Tratamentos (\%Ca) } \\
\cline { 2 - 5 } & $\mathrm{A}$ & $\mathrm{B}$ & $\mathrm{C}$ & $\mathrm{D}$ \\
\cline { 2 - 6 } & 0,40 & 0,07 & 0,26 & 0,48 \\
\hline Feno de Coast cross & 59,2150 & 16,1150 & 100,00 & 59,2620 \\
Farelo de milho & 26,7000 & 50,0000 & - & 26,7000 \\
Farelo de trigo & 4,0000 & 33,3400 & - & 4,0000 \\
Farelo de soja & 9,3000 & - & - & 9,3000 \\
Carbonato de cálcio, (p.a) & 0,5280 & - & - & 0,7380 \\
Fosfato monosódico, (p.a) & 0,2570 & 0,5450 & - & - \\
\hline Total & 100,0000 & 100,0000 & 100,0000 & 100,0000
\end{tabular}

Análise calculada (\%)

Matéria seca

$88,7600 \quad 88,0207 \quad 89,3700 \quad 88,7560$

Proteína bruta

$10,0158 \quad 10,1738 \quad 4,6300 \quad 10,0180$

Fibra bruta

$25,0423 \quad 10,1505 \quad 39,5200 \quad 25,0609$

Extrato etéreo

$2,8838 \quad 3,1448 \quad 2,5000 \quad 2,8850$

Materia mineral

$3,9734 \quad 2,6494 \quad 4,9900 \quad 3,9758$

Extrativo não nitrogenado

46,0540

61,3726

$37,7300 \quad 46,0720$

Composição analisada (\%)

Cálcio

0,4000

0,0736

0,2600

0,4840

Fósforo

0,3000

0,5016

0,1600

0,2338 


\section{Resultados e Discussão}

Após centrifugação de alíquota as amostras de urina foram submetidas ao estudo de sedimentoscopia para análise da presença de presença de cristais e células de descamação do aparelho urinário, não sendo observada anormalidade e a flora presente nas amostras de urina foi considerada típica da espécie. A determinação diária do clearance de $\mathrm{Cr}$ não mostrou alteração do metabolismo ou disfunção renal dos animais por influência dos tratamentos durante a fase experimental. Os animais não mostraram sinais clínicos compatíveis com quadros de deficiência ou excesso de minerais na dieta. Os níveis de Ca nas formulações adotadas mostram diferença $(P<0,0001)$ entre os tratamentos estudados. O quadro geral de analise variância mostrou efeito $(P<0,05)$ significativo de tratamentos, não significativo $(P>0,05)$ para tempos e ausência de interação $(P>0,05)$ entre tempos e tratamentos (Tabela 2$)$.

Tabela 2 - Análise de variância para efeito dos tratamentos, tempos e interação tempo $\times$ tratamento.

\begin{tabular}{l|c|c|c|c|c}
\hline $\begin{array}{l}\text { Fonte de } \\
\text { variação }\end{array}$ & G.L & $\begin{array}{c}\text { Soma dos } \\
\text { quadrados }\end{array}$ & $\begin{array}{c}\text { Quadrado } \\
\text { médio }\end{array}$ & $\begin{array}{c}\text { Valor } \\
\text { de F }\end{array}$ & $\begin{array}{l}\text { Probabi- } \\
\text { lidade>F }\end{array}$ \\
\hline Modelo & 19 & 846,36 & 44,54 & 2,11 & 0,0114 \\
Resíduo & 80 & $1.691,91$ & 21,15 & & \\
\hline Total & 99 & $2.538,27$ & & & \\
\hline
\end{tabular}

\begin{tabular}{l|c|c|c|c|c}
\hline $\begin{array}{l}\text { Fonte de } \\
\text { variação }\end{array}$ & G.L & Erro tipo I & $\begin{array}{c}\text { Quadrado } \\
\text { médio }\end{array}$ & $\begin{array}{c}\text { Valor } \\
\text { de } F\end{array}$ & Pr $>\mathrm{F}$ \\
\hline Tempo (T) & 4 & 65,41 & 16,35 & 0,77 & 0,54 \\
$\begin{array}{l}\text { Tratamento } \\
\text { (TRATA) }\end{array}$ & 3 & 417,03 & 139,01 & 6,57 & 0,0005 \\
T x TRATA & 12 & 363,93 & 30,33 & 1,43 & 0,1681 \\
\hline
\end{tabular}


Entre os tempos estudados, as médias do tratamento A para \% DRCr- Ca variaram de: 0: 3,52\%; 48: 5,94\%; 72: 5,27\%; 96: 10,15\%; e 120 horas: $7,77 \%$. As médias para \% DRCr- Ca entre os tratamentos estudados no tempo 0 horas foram de: A: 3,52\%; B: 5,10\%; C: 3,50\%; e D: 6,89\%. A análise conjunta das médias dos tratamentos nos tempos os \% DRCr- Ca de 6,53\% (A) e 6,34\% (D) diferiram $(P<0,05)$ das menores médias de 2,90\% (C) e 1,99\% ( $B, P<0,01)$ (Tabelas 3 e 4; Figura 1). A variação de médias do \% DRCr- Ca de 3,50\% a 6,89\% entre tratamentos (0 h) foram de 3,52\% (0 h) a 10,14\% (96 h) entre tempos do tratamento A são semelhante aos valores de médias para o \% DRCr- Ca reportados por Caple et al. (1982a, b) ao consideraram que eqüinos adultos recebendo dieta contendo 3,9 g de $\mathrm{Ca} / \mathrm{kg}$ de ração mostraram valor médio acima de 2,5\% para o \%DRCr- Ca.

As médias do \% DRCr- Ca obtidas nesta pesquisa refletem a biodisponibilidade esperada para o Ca presente nos ingredientes da dieta no tempo inicial (4 g Ca/kg MS ração) quando comparada à variação de $45 \%$ a $70 \%$ de disgestibilidade verdadeira do $\mathrm{Ca}$ presente nos ingredientes citados por Schryver et al. (1970a) ou acima de 65\% de digestibilidade verdadeira dos vários ingredientes utilizados na dieta de eqüinos apresentados por Hintz \& Schryver (1972, 1976). Portanto, nesta pesquisa as médias do \% DRCr- Ca dos tratamentos $(0 \mathrm{~h})$ e dos tempos do tratamento A presume-se balanço positivo de $\mathrm{Ca}$, predito pela excreção urinária média variando, respectivamente, de 25,94 $\mathrm{mg}(A)$ a $36,52 \mathrm{mg}$ (D) e 32,69 mg (grupo $A$, análise conjunta, Tabela 4$)$ de $C a$ na urina, contrapondo-se $(P<0,05)$ entre tempos ao grupo B com médias entre 2,57 mg (72 h) e 11,43 mg (96 h) e entre tratamentos pela análise conjunta dos dados $(P<0,01)$ com média de $12,75 \mathrm{mg} / \mathrm{dL}$ (grupo B - 0,74 g Ca/Kg MS ração) de Ca na urina (Tabelas 3 e 4). Estes resultados concordam com Schryver et al. (1970) ao reportarem valor de 5 a 6 mg/kg PV/dia de Ca na urina como perda obrigatória em cavalos com dieta de Ca calculada para mantença (retenção zero).

Nas situações de balanço negativo de Ca é gerado déficit no pool das reservas determinado por maior remoção que deposição para manutenção do 
fluído extracelular corpóreo constante, que pode ser acentuado em categorias com necessidades, alem de mantença, de crescimento e trabalho. Segundo os autores acima, as médias de excreções endógenas fecal e urinária foram de 20 $\mathrm{mg}$ e $5 \mathrm{mg} / \mathrm{kg} \mathrm{PV} /$ dia, respectivamente.

Assumindo, variação de absorção de $45 \%$ a $70 \%$ e média de $50 \%$ de digestibilidade verdadeira dos alimentos, segundo Schryver et al.(1974a), um pônei requer entre $35 \mathrm{mg}$ e $60 \mathrm{mg} \mathrm{Ca/kg} \mathrm{PV/dia} \mathrm{e} \mathrm{média} \mathrm{de} \mathrm{aproximadamente}$ $43 \mathrm{mg}$ de $\mathrm{Ca} / \mathrm{kg} \mathrm{PV} /$ dia para balancear perda obrigatória (mantença). Os autores acima concluíram que semelhante estimativa é obtida pela regressão da retenção $(Y)$ sobre a ingestão $(X), Y=0,393 X-16,86$ e coeficiente de correlação de 0,943 , a qual predita que a retenção é zero quando a ingestão é $16,86 \mathrm{~g} \mathrm{Ca} /$ dia.

Entretanto, visto as possíveis variações no aproveitamento dos nutrientes digestíveis totais da dieta, resultados desfavoráveis de avaliações de quadros clínicos devem ser interpretados levando-se em consideração a possível existência de complexadores com o $\mathrm{Ca}$, os fitatos e oxalatos, na formulação de rações baseando-se em digestibilidade verdadeira dos ingredientes. $\mathrm{Na}$ presente pesquisa foi calculada ingestão de níveis normais de $0,40 \%$ de cálcio para potras em crescimento de 18 a 24 meses de idade de acordo com a tabela de exigências preconizadas pelo NRC (1978), ou seja, ingestão diária de $24 \mathrm{~g}$ de cálcio. Com estes níveis de ingestão observou-se variação de médias entre tempos do grupo controle $(A)$ de 3,52\% ( 0 h) a 10,14\% (96 h) e entre tratamentos (0 h) de 3,50\% (C) a 6,89\% (D) para o \% DRCr- Ca, verificandose concordância nos dados obtidos neste experimento com aqueles citados na literatura para valores de \%DRCr-Ca maior que 2,50\% quando animais receberam dieta com $0,40 \%$ de $\mathrm{Ca}$ encontrados em dois experimentos realizados por Caple et al. (1982a, b).

Schryver et al. (1970a) relataram a existência de perda obrigatória de 2,5 $\mathrm{g}$ de $\mathrm{Ca} / 100 \mathrm{~kg}$ peso vivo por dia, sendo necessária a ingestão de $5,0 \mathrm{~g}$ de $\mathrm{Ca} / 100 \mathrm{~kg}$ de $\mathrm{PV} /$ dia considerando absorção média de 50\%. A retenção e excreção são diretamente proporcionais à ingestão apresentando coeficiente de 
correlação de 0,94 em função da ingestão para retenção e de 0,90 em relação à ingestão para a excreção urinária. De acordo com Schryver et al. (1974a), os rins dos eqüinos de 18 a 24 meses de idade excretam de $40 \%$ a $50 \%$ do $\mathrm{Ca}$ absorvido em dietas com teores de $1,50 \%$ de $\mathrm{Ca}$, confirmada pela grande quantidade de cristais de carbonato de Ca encontrada na urina realçando a importância dos rins na homeostasia do cálcio nesta espécie. Os autores acima afirmaram ainda que, para estágio equivalente de maturidade e ingestão de cálcio por ovinos e bovinos, eqüinos e pôneis absorvem mais Ca e excretam na urina maior proporção do Ca absorvido. Com 39,9\% de absorção, o duodeno é o principal sítio de absorção de Ca nos eqüinos. Segundo Schryver et al. (1970b) e Van Doorn et al. (2004a) na dieta equilibrada em Ca e P não há interferência do P na absorção do Ca.

No presente estudo, o ácido oxálico total determinado no feno de Coast cross foi inferior a $0,50 \%$ na MS da forragem. Teores de ácido oxálico acima de $0,50 \%$ na dieta podem interferir na absorção ao formarem sais insolúveis por complexação com o $\mathrm{Ca}$ ou ao serem absorvidos complexarem com o $\mathrm{Ca}$ sangüíneo (Swartzman et al., 1978). Em face da presença de fatores antinutricionais adversos e possíveis interações desfavoráveis ou antagonismos de macro e microminerais tem levado autores (Caple et al., 1982a, b; Mason et al. , 1988) a utilizarem além da análise bromatológica dos alimentos, avaliação da função renal através da determinação do clearance de creatinina para o íon Ca como prova bioquímica diagnóstica complementar capazes de avaliar o status do metabolismo do Ca. A técnica utilizada por vários autores foi baseada em trabalhos realizados por Knudsen (1959) que através da determinação do \% DRCr- paralelamente ao $\mathrm{DRCr}$ - do íon eletrolítico em questão (\% DRCr- $\mathrm{X}$ ) foi considerada de valor diagnóstico. No caso específico do íon $\mathrm{Ca}$, a padronização da dieta, ou seja, quantificação dos teores de Ca na dieta final, conjuntamente aos resultados do \% DRCr- Ca nesta pesquisa permitiu interpretação dos resultados e análise comparativa aos valores de literatura. 
As médias do tratamento B para \% DRCr-Ca nos tempos (h) variaram de: 0: 5,10\%; 48: 1,22\%; 72: 0,79\%; 96: 0,98\%; e 120 horas: 1,62\%. A maior média, $5,10 \%(0)$ mostrou diferença $(P<0,05)$ em relação às médias decrescentes nos tempos analisados (Tabela 3; Figura 1). Entre tratamentos, a menor média de $0,79 \%$ (B) obtida no tempo de 72 horas diferiu $(P<0,05)$ significativamente das médias de 5,27\% (A) e 5,96\% (D). A análise conjunta da variação das médias dos tempos entre tratamentos mostrou decréscimo significativo, sendo que a menor média de $1,94 \%$ diferiu $(P<0,01)$ em relação as maiores médias de 6,53\% (A), 6,34\% (D) para o \% DRCr-Ca (Tabela 4). No presente estudo, no tratamento $B$ o decréscimo $(\mathrm{P}<0,05)$ do $\% \mathrm{DRCr}-\mathrm{Ca}$ para $0,79 \%(72 \mathrm{~h})$ quando comparado aos demais tratamentos evidenciou-se que o baixo teor de $\mathrm{Ca}(\mathrm{B})$ na ração foi agravado pelo alto $\mathrm{P}$ na dieta. Entre tempos do grupo $B$, o decréscimo das médias foi significativo $(P<0,05)$ a partir de 48 h. Entretanto, Caple et al. (1982a, b) tem considerado o tempo de 72 h o que melhor reflete tais desequilíbrios, ou seja, na situação descrita acima, excesso de $\mathrm{P}$ e baixo Ca na ração e foi mencionado pelos autores acima como o referencial para monitorar dieta rica em grão e pobre em $\mathrm{Ca}$ influenciando médias do $\%$ DRCr-Ca menores que 2,5\%.

Nesta pesquisa, com $0,74 \mathrm{~g} \mathrm{Ca} / \mathrm{kg}$ MS na ração, entre tempos, a excreção urinária média de cálcio no tratamento $B$ decresceu significativamente $(\mathrm{P}<0,05)$ de $31,23 \mathrm{mg}(0 \mathrm{~h})$ para $2,57 \mathrm{mg} / \mathrm{dL}(72 \mathrm{~h})$ de Ca na urina, valores estes de médias semelhantes aos reportados por Schryver et al. (1970a) utilizando $1,5 \mathrm{~g} \mathrm{Ca} / \mathrm{kg}$ da dieta na alimentação de pôneis, sendo descrito pelos autores, balanço negativo de Ca com excreção endógena urinária de 5 a 6 mg/kg PV/dia em pôneis (Tabela 3; Figura 1). Schryver \& Hintz (1972) e Schryver et al. (1971) mencionaram que não houve influência de teores aumentados de Ca na dieta sobre a absorção do $\mathrm{P}$, uma vez que os principais sítios de absorção de P são a segunda metade do intestino delgado, colo dorsal e pequeno colo, mas situação contrária sim, o que torna o cavalo ainda mais vulnerável ao excesso de $\mathrm{P}$ na dieta. Nesta pesquisa, no tratamento $\mathrm{B}$, baixo Ca e alto $P$, com fontes ricas em $P$ (50\% milho, 33,34\% de farelo de trigo e 
fonte suplementar P) apresentou excreção diminuida de $\mathrm{Ca}$ na urina concordando Van Doorn et al. (2004a,b).

Tabela 3 - Valores médios do \% DRCR- Ca entre os tempos e tratamentos estudados em cinco potros da raça árabe e mestiça árabe

\begin{tabular}{|c|c|c|c|c|c|}
\hline \multirow{4}{*}{ Tratamentos } & \multicolumn{5}{|c|}{ Tempos (horas) } \\
\hline & 0 & 48 & 72 & 96 & 120 \\
\hline & \multicolumn{5}{|c|}{ Médias* \% DRCr-Ca } \\
\hline & \multicolumn{5}{|c|}{$5 \%$ de significância } \\
\hline$A$ & $3,52 a^{A^{* *}}$ & $5,95 a^{A}$ & $5,27 a^{A}$ & $10,14 a^{A}$ & $7,77 a^{A}$ \\
\hline B & $5,10 a^{A}$ & $1,22 b^{A}$ & $0,79_{b} B$ & $0,98 b^{A}$ & $1,62 b^{A}$ \\
\hline C & $3,50 a^{A}$ & $5,04 a^{A}$ & $1,43 b^{B}$ & $2,23 \mathrm{a}^{\mathrm{A}}$ & $2,30 a^{A}$ \\
\hline \multirow[t]{2}{*}{ D } & $6,89 a^{A}$ & $3,80_{a} A^{A}$ & $5,96 a^{A}$ & $3,71_{\mathrm{a}^{\mathrm{A}}}$ & $11,42 a^{A}$ \\
\hline & \multicolumn{5}{|c|}{$1 \%$ de significância } \\
\hline A & $3,52 a^{A}$ & $5,95 a^{A}$ & $5,27 a^{A B}$ & $10,14 a^{A}$ & $7,77 a^{A}$ \\
\hline B & $5,10 a^{A}$ & $1,22 b^{A}$ & $0,79 b^{B}$ & $0,98 b^{A}$ & $1,62 b^{A}$ \\
\hline C & $3,50 a^{A}$ & $5,04 a^{A}$ & $1,43 \mathrm{a}^{\mathrm{AB}}$ & $2,23 \mathrm{a}^{\mathrm{A}}$ & $2,31_{a^{A}}$ \\
\hline D & $6,89 a^{A}$ & $3,80_{a}{ }^{A}$ & $5,96 a^{A}$ & $3,71_{a^{A}}$ & $11,42 a^{A}$ \\
\hline
\end{tabular}

Ca na urina $(\mathrm{mg} / \mathrm{dL}), \mathrm{P}<0,05$

$\begin{array}{llllll}\mathrm{A} & 25,94 \mathrm{~b}^{\mathrm{A}} & 25,08 \mathrm{~b}^{\mathrm{A}} & 37,57 \mathrm{a}^{\mathrm{bA}} & 47,20 \mathrm{a}^{\mathrm{A}} & 27,64 \mathrm{ab}^{\mathrm{A}} \\ \mathrm{B} & 31,23 \mathrm{a}^{\mathrm{A}} & 8,46 \mathrm{~b}^{\mathrm{A}} & 2,57 \mathrm{~b}^{\mathrm{B}} & 11,43 \mathrm{~b}^{\mathrm{B}} & 10,03 \mathrm{~b}^{\mathrm{A}} \\ \mathrm{C} & 26,56 \mathrm{a}^{\mathrm{A}} & 22,28 \mathrm{a}^{\mathrm{A}} & 24,56 \mathrm{a}^{\mathrm{AB}} & 19,34 \mathrm{a}^{\mathrm{B}} & 36,86 \mathrm{a}^{\mathrm{A}} \\ \mathrm{D} & 36,52 \mathrm{a}^{\mathrm{A}} & 26,39 \mathrm{a}^{\mathrm{A}} & 37,03^{\mathrm{a}} & 26,80 \mathrm{a}^{\mathrm{AB}} & 32,53 \mathrm{a}^{\mathrm{A}}\end{array}$

*Médias de cinco repetições por tempo (0,48, 72, 96 e $120 \mathrm{~h})$

**Médias com letras distintas minúsculas na linha ou maiúsculas na coluna diferem entre si pelo teste de Tukey ao nível de significância indicado 
Experimentalmente, Argenzio et al. (1974) reportaram que na regulação da homeostase do Ca e $\mathrm{P}$, excesso de $\mathrm{P}$ e baixo Ca na dieta, levaram a hipersecreção da glândula paratireóide com aumento da reabsorção óssea podendo induzir generalizada descalcificação osteodistrófica e aparecimento do hiperparatiróidismo nutricional secundário (HPTNS), quando perdurarem alto teor de $\mathrm{P}$ na dieta associado ao baixo teor de $\mathrm{Ca}$. No presente experimento, para o \% DRCr- Ca pode ser observado decréscimo significativo $(P<0,01)$ entre todos os tempos do tratamento $B$ quando comparados à média de $5,10 \%$ $(0 \mathrm{~h})$. Entre tratamentos, no tempo de $72 \mathrm{~h}$, a menor média de $0,79 \%$ diferiu $(P<0,05)$ das médias de $5,27 \%$ (A) e $5,96 \%$ (D) para o \% DRCr-Ca com concomitante redução na excreção urinária de $\mathrm{Ca}$ dos respectivos tratamentos (Tabela 3; Figura 1).

Teeter et al. (1967) e Bourke (1968) examinaram o aparecimento de patologias ósseas em eqüinos, advindas da dieta desequilibrada com baixo $\mathrm{Ca}$ e alto $\mathrm{P}$ e obtiveram melhora do estado geral com regressão significativa sinais clínicos dos animais com correção dos níveis destes elementos na ração. Schryver et al. (1971) verificaram que altos teores de $\mathrm{P}$ na dieta $(1,20 \%$ de $\mathrm{P})$ prejudicavam a absorção do $\mathrm{Ca}$, determinando aumento na taxa de deposição e remoção do Ca ósseo e aumento da excreção de $\mathrm{P}$ na urina influenciado pelo paratormônio que age sobre os rins aumentando a excreção de $\mathrm{PO}_{4}=$ e diminuindo a excreção de Ca. Nordin \& Peacock (1969) e Gans \& Mercer (1984) reportaram o importante papel que os rins exercem sobre a homeostase do Ca reabsorvendo do filtrado na deficiência ou excretando quando no excesso do elemento.

Da mesma forma, Habener (1981) e Massry et al. (1973) ressaltaram a importância da manutenção do fluído extracelular constante através da regulação da absorção intestinal do $\mathrm{Ca}$, reabsorção tubular renal do Ca filtrado, aumento da excreção renal de $\mathrm{PO}_{4}=$ quando em excesso de $\mathrm{P}$ na dieta, ações estas de extrema importância na homeostase do Ca e P. Raisz \& Kream (1981) afirmaram que a manutenção na normalidade e ou desenvolvimento do esqueleto é depende do mecanismo de regulação da homeostasia dos 
elementos minerais em questão, concordando com Nordin (1958), Traver et al. (1976, 1977), Coffman (1980), Caple et al. (1982a) e Cuddeford et al. (1990) que as osteopatias caracterizadas por desequilíbrio de Ca e P na dieta, seja pela combinação de baixo $\mathrm{Ca}$ e alto $\mathrm{P}$, excesso de $\mathrm{Ca}$ e $\mathrm{P}$, Ca normal com excesso de $\mathrm{P}$ na dieta, podem ser diagnosticadas e prevenidas a tempo com a coleta simultaneamente de amostra de soro e urina para dosagem de $\mathrm{Ca}$ e $\mathrm{Cr}$ determinando-se o \% DRCr- Ca.

Sobre as anomalias ósseas clínicas e subclínicas resultantes de desequilíbrio de Ca na dieta, vários autores dedicaram importantes estudos sobre Ca e P na dieta. Krook (1968) classificou o HPTNS como sendo de natureza compensatória e induzido por hipocalcemia o que também foi reportado por Tennant et al. (1982) ao estudaram em seis cavalos portadores insuficiência renal crônica apresentando glomerulonefrite crônica e nefrite intersticial crônica associadas com hipercalcemia e hipofosfatemia resultantes da incapacidade renal para manutenção da homeostase do Ca e P.

As médias do tratamento C $(2,6 \mathrm{~g} / \mathrm{kg} \mathrm{MS})$ para \% DRCr-Ca nos tempos ( $\mathrm{h}$ ) variaram de: 0 : 3,50\%; 48: 5,04\%; 72: 1,43\%; 96: 2,23\%; e 120: 2,30\%. O decréscimo da média de 1,43\% (grupo $C, 72 \mathrm{~h}$ ) mostrou diferença $(P<0,05)$ em relação as médias de 5,27\% ( $A, 4 \mathrm{~g}$ de $\mathrm{Ca} / \mathrm{kg} M S)$ e 5,96\% ( $\mathrm{D}, 4,84 \mathrm{~g}$ $\mathrm{Ca} / \mathrm{kg} \mathrm{MS}$ ) (Tabela 3; Figura 1). Os resultados da presente pesquisa concordam com o reportado por Caple et al. (1982a, b) ao relatarem valores menores que 2,5\% quando a dieta era deficiente em $\mathrm{Ca}(1,8 \mathrm{~g} \mathrm{Ca})$ ou deficiente em $\mathrm{Ca}(2,4 \mathrm{~g} \mathrm{Ca})$ combinado com alto $\mathrm{P}(6,1 \mathrm{~g} \mathrm{P} / \mathrm{kg} \mathrm{MS})$ na dieta, o que também foi verificado no presente experimento na dieta do tratamento $B$, com baixo Ca $(0,74)$ e P alto $(5,02 \mathrm{~g} \mathrm{P} / \mathrm{kg}$ MS da ração). Nesta pesquisa, entre tratamentos, a menor média de 2,90\% (grupo C) para o \% DRCr-Ca obtida na análise conjunta dos dados diferiu $(P<0,05)$ em relação as maiores médias obtidas nos tratamentos A $(6,53 \%)$ e D $(6,34 \%)$ (Tabela 4$)$. 
Tabela 4 - Análise conjunta dos valores médios do \% DRCr- Ca e Ca e $\mathrm{Cr}$ no soro e urina de cinco potras medidos nos tempos $(0,48,72,96$ e $120 \mathrm{~h}$ ) e tratamentos estudados

\begin{tabular}{|c|c|c|c|c|}
\hline \multirow{3}{*}{ Determinações } & \multicolumn{4}{|c|}{ Tratamentos } \\
\hline & A & B & C & $\mathrm{D}$ \\
\hline & \multicolumn{4}{|c|}{ Médias* } \\
\hline \% DRCr-Ca & $6,53 a^{A^{* *}}$ & $1,94 b^{B}$ & $2,90_{b}{ }^{A B}$ & $6,34 a^{A}$ \\
\hline Ca no soro & $11,75^{A}$ & $11,49 a^{A}$ & $11,13^{A}{ }^{A}$ & $11,76_{a}^{A}$ \\
\hline Ca na urina & $32,69 a^{A}$ & $12,75 b^{B}$ & $25,93_{a} A B$ & $31,86_{a}{ }^{A}$ \\
\hline Cr no soro & $1,87 b^{A}$ & $1,99 a^{A}{ }^{A}$ & $2,09 a_{a}^{A}$ & $1,50 c^{B}$ \\
\hline $\mathrm{Cr}$ na urina & $123,26 b^{A B}$ & $141,62 a_{b}{ }^{A B}$ & $207,53 a^{A}$ & $97,00_{b} B$ \\
\hline
\end{tabular}

*Médias de vinte cinco repetições nos tempos $(0,48,72,96$ e $120 \mathrm{~h})$

**Médias com letras distintas minúsculas $(\mathrm{P}<0,05)$ ou maiúsculas $(P<0,01)$ na linha diferem entre si pelo teste de Tukey

Observações semelhantes foram obtidas por Schryver et al. (1970a) quando utilizaram $1,5 \mathrm{~g} \mathrm{Ca} / \mathrm{kg}$ MS na dieta de pôneis e constataram balanço negativo de Ca com decréscimo na excreção renal do íon. As médias para \% DRCr- Ca no tratamento D nos tempos (horas) variaram de 3,71\% (96 h menor média) a 11,42\% (120 h), entretanto, as diferenças $(P>0,05)$ entre tempos não foram significativas (Tabela 3; Figura 1). Entre tratamentos, no tempo de 72 horas a maior média de 5,96\% (grupo $D$ ) diferiu $(P<0,05)$ das menores médias de 0,79\% (grupo B) e 1,43\% (grupo C) para o \% DRCr- Ca. Os resultados da presente pesquisa são corcordantes com os achados de Knight et al. (1985) ao estudaram a incidência das enfermidades ósseas em cavalos e sua correlação com a dieta mineral, enumerando as manifestações clínicas de acordo com a osteopatia apresentada. A osteodistrofia generalizada vista nos processos de descalcificação, segundos os autores acima foram causadas por dietas desequilibradas em Ca e P. 
Carvalho, P.R. d'Arce, R.D., Machado Neto, R., et al. Aplicação da técnica da taxa de depuração renal de creatinina para avaliação do "status" do cálcio em eqüinos. PUBVET, Londrina, V. 2, N. 44, Art\#428, Nov1, 2008.

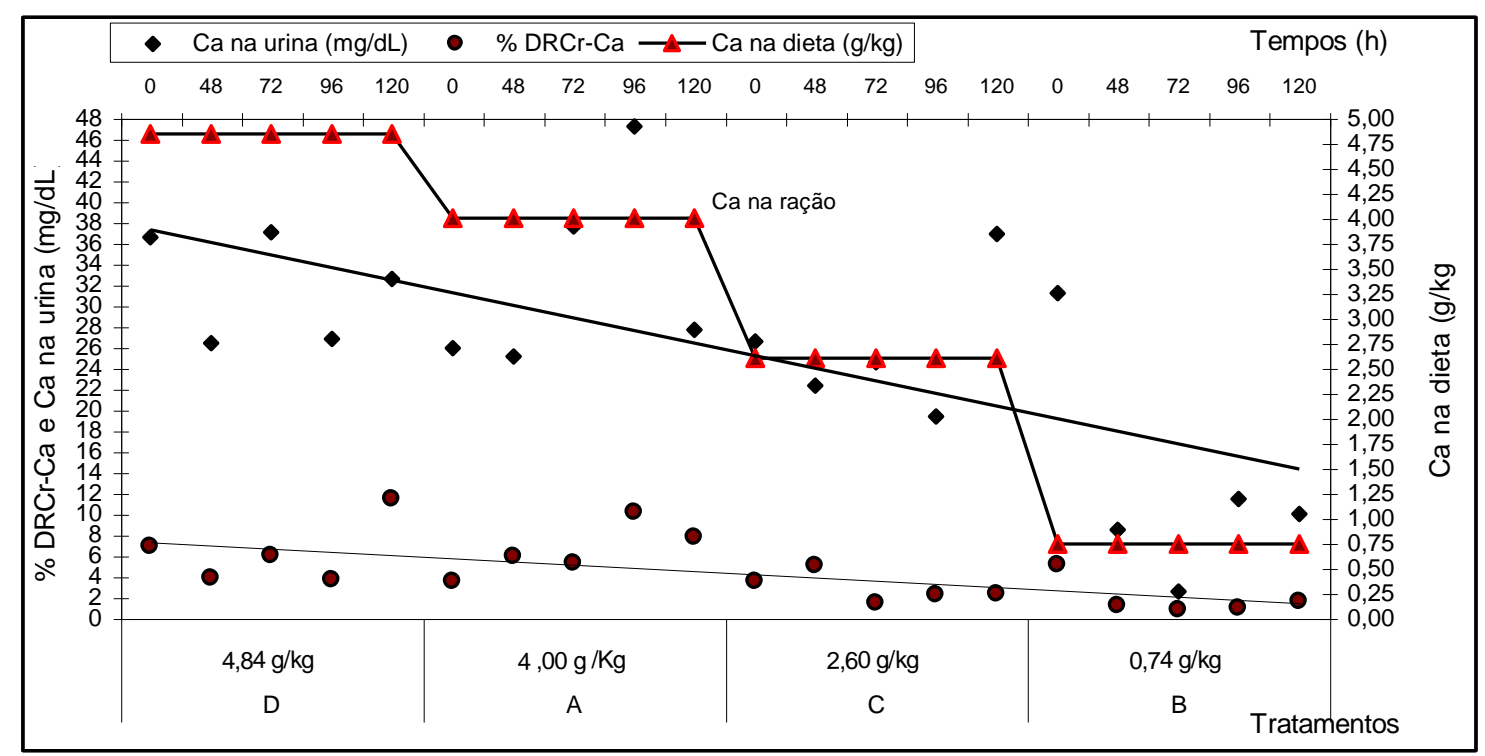

Figura 1 - Valores médios de Ca na urina e do \% DRCr-Ca de acordo com os tratamentos nos tempos de $0,48,72,96$ e 120 horas

Ainda, os autores acima determinaram em 19 potros de um ano de idade, correlação positiva $(r=0,71)$ entre os teores dos constituintes da ração total e o aparecimento de patologias ósseas $(P<0,0001)$, ou seja, quanto menor o erro na ração para níveis usados entre $0,50 \%$ a $1,37 \%$ de $\mathrm{Ca}$, menor o número de problemas ósseos. Da mesma forma, Thatcher (1991) relacionou o desenvolvimento de doenças ortopédicas no cavalo e os teores de nutrientes na dieta quando foi possível estabelecer relação direta entre o aparecimento de osteopatias e os níveis deficientes de Ca na ração e Hintz et al. (1979) correlacionaram positivamente o efeito de dietas desequilibradas ou deficientes em Ca de éguas gestantes e o nascimento de potros com anomalias ósseas. 
$\mathrm{Na}$ análise conjunta dos dados, as rações contendo $0,48 \%$ de $\mathrm{Ca}$ (grupo $\mathrm{D}$ - cálcio alto) e 0,40\% de Ca (grupo A - cálcio normal) diferiram $(P<0,05)$ da ração contendo $0,26 \%$ de Ca (grupo $C$ - Ca deficiente) e da ração $(P<0,01)$ contendo $0,074 \%$ de $\mathrm{Ca}$ (grupo B - Ca baixo) na dieta das potras em crescimento, influenciaram as médias do \% DRCr- Ca, respectivamente, de $6,34 \%$ (D), 6,53\% (A), 2,90\% (C) e 1,94\% (B) (Tabela 4; Figura 1). Resultados semelhantes a presente pesquisa, entretanto, médias maiores do \%DRCr- Ca foram apresentados por Cuddeford et al. (1970) ao reportarem que com teores de $11,4 \mathrm{~g} \mathrm{Ca} / \mathrm{kg}, 19,0 \mathrm{~g} \mathrm{Ca} / \mathrm{kg}$ e 26,3 g Ca/kg M.S do feno, obtiveram \% DRCr- Ca de 25\%, 29\% e 33\%, respectivamente. Nesta pesquisa, o tratamento $\mathrm{D}$ com 4,84 g Ca/kg de MS atingiu 11,42\% (120 h maior média) (Tabela 3; Figura 1).

A média geral dos tratamentos foi de $30,06 \mathrm{mg} / \mathrm{dL}(0 \mathrm{~h})$ de Ca na urina. Entre tratamentos, as diferenças $(P>0,05)$ entre médias $(0 h)$ não foram significativas (Tabela 3; Figura 2). As médias de $\mathrm{Ca}(\mathrm{mg} / \mathrm{dL})$ na urina entre tempos do tratamento B variaram de 2,57 mg (72 h) a $11,43 \mathrm{mg} \mathrm{Ca} / \mathrm{dL}(96 \mathrm{~h}$ ) na urina, representando decréscimo significativo $(P<0,01)$ de $91,60 \%$ e $63,40 \%$, respectivamente, em relação à média de $31,23 \mathrm{mg} \mathrm{Ca} / \mathrm{dL}(\mathrm{B}-0 \mathrm{~h}) \mathrm{na}$ urina.

Entre tratamentos $(96 \mathrm{~h})$ diferença $(P<0,05)$ significativa foi mantida entre as médias de $11,43 \mathrm{mg}$ (grupo $B$ ), e a maior média de $47,20 \mathrm{mg} / \mathrm{dL}$ (grupo A) de Ca na urina. A análise conjunta dos dados para os tempos entre tratamentos mostrou diferença $(P<0,05)$ para a média de $12,75 \mathrm{mg}$ (B) em relação as maiores médias de 32,69 $\mathrm{mg}(A), 31,86$ (D) e 25,93 mg/dL (C) de Ca na urina, sendo que diferença $(P<0,01)$ significativa foi mantida para os tratamentos A e D em relação ao grupo (Tabela 4). Parâmetros semelhantes de excreção de Ca na urina foram testemunhados por Schryver et al. (1970a) ao reportarem que em pôneis em crescimento os teores crescentes de Ca de 0,15\% (baixo), 0,80\% (intermediário) e 1,50\% (alto) na dieta determinaram médias de excreção urinária proporcionalmente crescente a quantia de $\mathrm{Ca}$ ingerida, respectivamente, de 5,5 mg, $63 \mathrm{mg}$ e $99 \mathrm{mg} / \mathrm{kg} \mathrm{PV} /$ dia de excreção. 
Na presente pesquisa, na análise conjunta dos tempos de cada tratamento, as médias de 32,69 mg (grupo A, 0,40\% Ca) e 31,86 mg/dL (grupo D - 0,48\% Ca) na urina diferiu $(\mathrm{P}<0,01)$ da média de $12,75 \mathrm{mg} / \mathrm{dL}$ (grupo $\mathrm{B}-0,074 \% \mathrm{Ca}$ ) de Ca na urina. Entre tratamentos, as proporções de Ca na ração e na urina mostraram regressão linear significativa $\left(Y=0,8216 X+10,7890 ; R^{2}=\right.$ 0,9179 ), significando que maiores excreções de Ca (grupos A e D) e menores excreções (grupo B e C) de Ca na urina foram dependes dos níveis de ingestões de Ca na dieta dos animais (Figura 2). Estas observações estão de acordo com Schryver et al. (1970a) ao relatarem que pôneis alimentados com três diferentes teores de 0,15\% Ca (baixo), 0,80\% Ca (intermediário) e 1,50\% (alto) de Ca na dieta apresentaram, respectivamente, médias de 0,55 g Ca, 2,1 g Ca e 3,3 g Ca/100 kg PV/dia de excreção urinária.

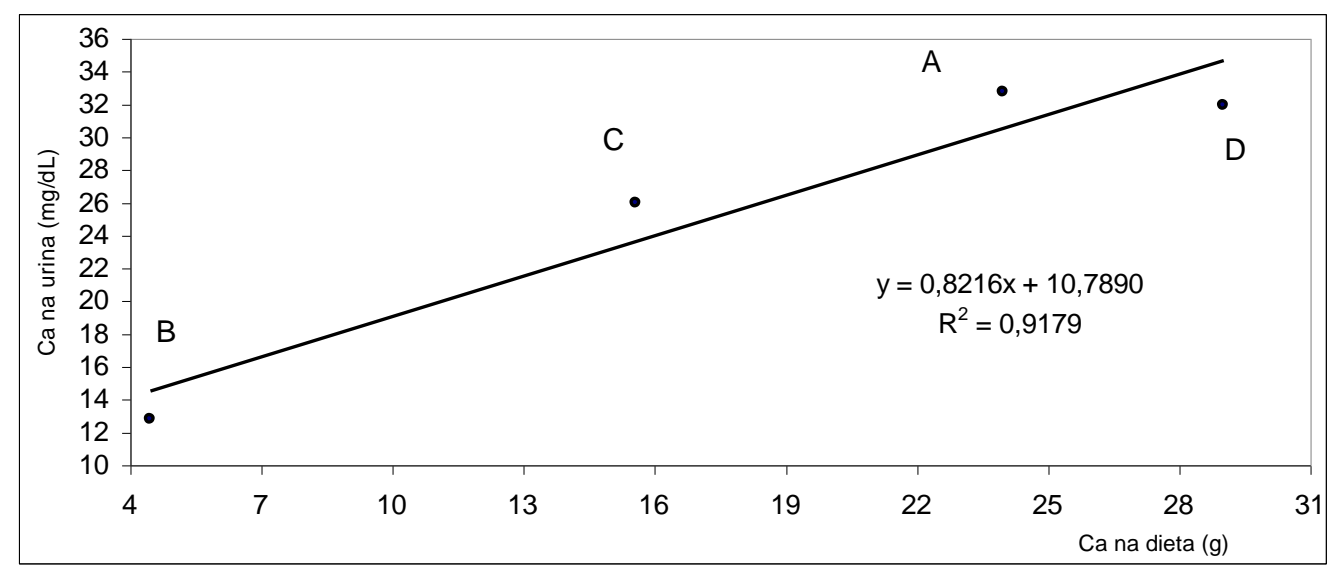

Figura 2 - Valores médios de Ca na urina $(\mathrm{mg} / \mathrm{dL})$ em função dos teores de cálcio na ração $(\mathrm{g})$ expresso pela regressão linear $Y=0,8216 \mathrm{X}+10,7890$, segundo os tratamentos estudados

Os autores acima encontraram coeficiente de correlação de 0,91 entre o $\mathrm{Ca}$ absorvido e excretado na urina e afirmaram ainda que os pôneis com $\mathrm{Ca}$ alto na dieta excretaram $25 \%$ do Ca em 24 h e $35 \%$ em 10 dias e os mesmos animais excretaram $2,5 \%$ de $\mathrm{Ca}$ em 24 h e 3,8\% do Ca em 10 dias. Tais dados obtidos neste estudo demonstraram a importância da determinação do \% 
DRCr- Ca realizado na presente pesquisa, face ao rápido ajuste pelo mecanismo de feed back sobre a regulação do Ca plasmático as expensas do pool de reservas e o efeito negativo ao esqueleto dos níveis excessivos de $\mathrm{P}$ na dieta (Whitlock et al. 1970).

Confirmando as observações dos autores acima, nesta pesquisa dieta desequilibrada contendo baixo $\mathrm{Ca}$ alto $\mathrm{P}$ (B) mostraram resultados semelhantes aos obtidos por Schryver et al. (1970a) ao reportarem valor de 5 a $6 \mathrm{mg} / \mathrm{kg}$ PV/dia de Ca na urina de cavalos. Nestas situações a homeostasia mantem níveis constantes no fluído extracelular e balanço negativo de Ca é observado face ao deficit gerado no pool de reservas em categorias de animais com necessidades adicionais, alem da mantença, para crescimento e trabalho.

\section{Conclusões}

Os dados obtidos no presente experimento permitem concluir que: O \% DRCr-Ca é índice que detectou com eficiência no tempo quando a dieta de eqüinos foi extremamente deficiente em $\mathrm{Ca}$ e teve excesso de $\mathrm{P}$, sendo que a partir de $48 \mathrm{~h}$ ou $72 \mathrm{~h}$ é possível averiguar no tempo o status mineral e metabólico do $\mathrm{Ca}$ em eqüinos. Eqüinos alimentados com alto $\mathrm{Ca}$ na ração no tratamento $D$ excretaram mais $C a$ na urina no tempo quando comparados a animais com dieta pobre em Ca no tratamentos $\mathrm{C}$ e ou dieta com excesso de $\mathrm{P}$ no tratamento B. Animais que apresentaram o \% DRCr-Ca acima de 3,50\% provinham de dieta com níveis normais de $\mathrm{Ca}$ e foram considerados o valor mínimo para o \% $\mathrm{DRCr}-\mathrm{Ca}$. Dieta com baixo $\mathrm{Ca}$ e alto $\mathrm{P}$ revelaram redução significativa no \%DRCR-Ca de $5,10 \%$ para $1,22 \%$ em 48 h após administração da dieta com Ca e P normal mostrando a eficiência da técnica na deteç̧ão precoce nas dietas com níveis desequilibrados de Ca na ração diária dos animais. Os valores médios de cálcio analisados no soro dos eqüinos não expressaram o desequilíbrio de cálcio na dieta, exceto transitoriamente, em determinado tempo por influência do tratamento, denotando a eficácia dos mecanismos homeostáticos na regulação da mantença da composição do fluído 
Carvalho, P.R. d'Arce, R.D., Machado Neto, R., et al. Aplicação da técnica da taxa de depuração renal de creatinina para avaliação do "status" do cálcio em eqüinos. PUBVET, Londrina, V. 2, N. 44, Art\#428, Nov1, 2008.

extracelular. Antes e durante a fase experimental, os animais avaliados para a função renal através da urinálise e clearance de creatinina não mostraram disfunção renal por influência dos tratamentos no período estudado.

\section{Referências Bibliográficas}

ARGENZIO, R.A.; LOWE, J.E.; HINTZ, H.F. Calcium and phosphorus homeostasia in horses. Journal of Nutrition, v.104, n.1, p. 18-27, 1974.

ASSOCIATION OF OFFICIAL ANALYTICAL CHEMISTS - AOAC. Official methods of analysis of the association of official analytical chemists. 11.ed. Washington, D.C. 1970. 1015p.

BLANEY, B.J.; GARTNER, R.J.W.; McKENZIE, R.A. The effects os oxalate in some tropical grasses on the availability to horses of calcium, phosphorus and magnesium. Journal of Agricultural Science, v.97, n.3, p.507-514, 1981.

BOURKE, J.M. Feeding of thoroughbred horses. Australian Veterinary Journal, v.44, n.5, p. 241-245, 1968.

BROBST, D.F.; LEE, H.A.; SPENCER, G.R. Hipercalcemia and hipophosphatemia in a Mare with renal insufficiency. Journal of the American Veterinary Association, v. 173, n.10, p. $1370-1372,1978$.

CAPLE, I.W.; BOURNE, J.M.; ELLIS, P.G. An examination of the calcium and phosphorus nutrition of throughbred race horses. Australian Veterinary Journal, v. 58, n.4, p.132135, $1982 b$.

CAPLE, I.W.; DOAKE, P.A.; ELLIS, P.G. Assessment of the calcium and phosphorus nutrition in horses by analysis of urine. Australian Veterinary Journal, v.58, n.4, p. 125-31, 1982a.

COFFMAN, J. Percent creatinine clearance ratios. Veterinary Medicine Small and Animal Clinician, v.75, n.4, p.671-676, 1980.

CUDDEFORD, D.; WOODHEAD, A.; MUIRHEAD, R. Potencial of alfalfa as a source of calcium for calcium deficient horses. Veterinary Record, v.126, n.17, p.425-429, 1990.

CYMBALUK, N.F.; CHRISTENSEN, D.A. Nutrient utilization of pelleted and unpelleted forages by ponies. Canadian Journal of Animal Science, Ottawa, v.66, n.2, p.237-244, 1986.

ELFERS, R.S.; BAYLY, W.M.; BROBST, D.F. et al. Alterations ins calcium, phosphorus and Cterminal parathyroid hormone levels ins equine acute renal disease. Cornell Veterinarian, v.76, p.317-329, 1986.

GANS, J.H.; MERCER, P.F. In: SWENSON, M.J. (Ed.). Duke's Physiology of domestic animals. The kidneys. Cornell University Press Ltd., London, Tenth edition, 1984. p. 507-536.

HABENER, J.F. Regulation of parathyroid hormone secretion and biosynthesis. Annual Review of Physiology, v.43, p.211-223, 1981.

HANDA, S.P.; MORRIN, P.A. Diagnostic indices in acute renal failure. Canadian Medical Association Journal, v.96, n.2, p.78-82, 1967.

HINTZ, H.F.; SCHRYVER, H.F. Availability to ponies of calcium and phosphorus from various supplements. Journal of Animal Science, v.34, n.6, p. 979-980, 1972.

HINTZ, H.F.; SCHRYVER, H.F. Nutrition and bone development in horses. Journal of the American Veterinary Medical Association, v.168, n.1, p. 39-44, 1976.

HINTZ H.F; HINTZ R.L; VAN VLECK, L.D. Growth rate of thoroughbreds, effect of age of dam, year and month of birth, and sex of foal, Journal of Animal Science, v. 48, n.3, p. 480-487, 1979.

KNIGHT, D.A.; GABEL, A.A.; REED, S.M. et al. Correlation of dietary mineral to incidence and severity of metabolic bone disease in Ohio and Kentuck. In: Annual Convention of the American Association Equine Practit, 31., KANSAS, 1985. Proceedings... Lexington, Ohio State University, 1985, p. 445-461. 
Carvalho, P.R. d'Arce, R.D., Machado Neto, R., et al. Aplicação da técnica da taxa de depuração renal de creatinina para avaliação do "status" do cálcio em eqüinos. PUBVET, Londrina, V. 2, N. 44, Art\#428, Nov1, 2008.

KNUDSEN, E. Renal clearance studies on the horse. I: inulin, endogenous creatinine and urea. Acta Veterinaria Scandinavica, v.1, p.52-66, 1959.

KROOK, L. Dietary calcium-phosphorus and lameness in the horse. Cornell Veterinarian, v.58, p.58-73, 1968. Supplement.

LANE, V.M.; MERRITT, A.M. Reliability of single-sample phosphorus fractional excretion determination as a measure of daily phosphorus renal clearance in equids. American Journal of Veterinary Research, v.44, n.3, p. 500-502, 1983.

MASON, D.K.; WATKINS, K.L.; MCNIE, J.T. Diagnosis, treatment and prevention of nutritional secondary hyperparathyroidism in throughbred race horses in Hong kong. Equine Practice, v.10, n.3, p.10-17, 1988.

MASSRY, S.G.I.; FRIENER, R.R.M.; COBURN, J.W. Excretion of phosphate and calcium. Archives of Internal Medicine, v.131, n.6, p.828-859, 1973.

MORRIS, D.D.; DIVERS, T.J.; WHITLOCK, R.H. Renal clearance and fractional excretion of electrolytes over a 24-hours period in horses. American Journal of Veterinary Research, v.45, n.11, p.2431-2435, 1984.

NATIONAL RESAEARCH COUNCIL - NRC. Nutrient Requeriments of Domestic Animals, 6. Nutrient requeriments of horses. Washington, D.C.: National Academy of Sciences, 1978. 33p.

NORDIN, B.E.; PEACOCK, M. Role of kidney in regulation of plasma calcium. Lancet, v.13, n.2, p.1280-1283, 1969.

NORDIN, B.E. Primary and secondary hyperparathyroidism. Advances Internal Medicine, v.9, p.81-105, 1958.

RAISZ, L.G.; KREAM, B.E. Hormonal control of skeletal, growth. Annual Review of Physiology, v.43, p.225-238, 1981.

SAS Institute. SAS $\square / S T A T$. User's Guide: statistical version. SAS Institute, 1994.

SCHRYVER, H.F.; CRAIG, P.H.; HINTZ, H.F. Calcium metabolism in ponies fed varying levels of calcium. Journal of Nutrition, v.100, n.8, p.955-964, 1970a.

SCHRYVER, H.F.; CRAIG, P.H.; HINTZ, H.F. et al. The site of calcium absortion in the horse. Journal of Nutrition, v.100, n.10, p.1127-1132, 1970b.

SCHRYVER, H.F.; HINTZ, H.F.; CRAIG, P.H. Calcium metabolism in ponies fed high phosphorus diet. Journal of Nutrition, v.101, n.2, p.259-264, 1971.

SCHRYVER, H. F.; HINTZ, H.F. Calcium and phosphorus requeriments of the horse: A review. Feedstuffs, v.44, p.35-36, 38, 1972.

SCHRYVER, H.F.; HINTZ, H.F.; LOWE, J.E. Calcium and phosphorus in the nutrition of the horse. Cornell Veterinarian, v.64, n.4, p.493-515, 1974 a.

SCHRYVER, H.F.; HINTZ, H.F.; LOWE, J.E. et al. Mineral composition of young horses. Journal of Nutrition, v.104, n.1, p.124-132, 1974b.

SWARTZMAN, J.A.; HINTZ, H.F.; SCHRYVER, H.F. Inhibition of calcium absortion in ponies fed diets containing oxalic acid. American Journal of Veterinary Research, v.39, n.10, p.1621-1623, 1978.

TEETER, S.M.; STILLIONS, M.C.; NELSON, W.E. Maintenance levels of calcium and phosphorus in horses. Journal of the American Veterinary Medical Association Journal, V.151, n.12, p.1625-1628, 1967.

TENNANT, B.; BETTLEHEIM, P.; KANEKO, J.J. Paradoxic hypercalcemia and hypophosphatemia associated with chronic renal failure in horses. Journal of the American Veterinary Medical Association, v.180, n.6, p.630-634, 1982.

THATCHER, C.D. Nutritional aspects of development orthopedic disease in growing horses. Veterinary Medicine, v.7, p.743-747, 1991.

TRAVER, D.S.; COFFMAN, J.R.; MOORE, J.N. et al. Urine clearance ratios as diagnostic acid in equine metabolic disease. Proceedings of the American Association of Equine Practitioners... St. Louis, v.22, p.177-183, 1976.

TRAVER, D.S.; SALEN, C.; COFFMAN, J.R. et al. Renal metabolism of endogenous substances in the horse: volumetric vs clearance ratio methods. Journal of Equine Medicine and Surgery, v.1, n.11, p. 378-382, 1977. 
Carvalho, P.R. d'Arce, R.D., Machado Neto, R., et al. Aplicação da técnica da taxa de depuração renal de creatinina para avaliação do "status" do cálcio em eqüinos. PUBVET, Londrina, V. 2, N. 44, Art\#428, Nov1, 2008.

VAN DOORN, D.A.; EVERTS, H.; WOUTERSE, H.; BEYNEN, A.C. The apparent digestibility of phytate phosphorus and the influence of supplemental phytase in horses. Journal of Animal Science, v.82, n.6, p.1756-1763, 2004a.

VAN DOORN, D.A.; VAN DER SPER, M.E.; EVERTS, H.; WOUTERSE, H.; BEYNEN, A.C. The influence of calcium intake on phosphorus digestibility in mature ponies. Journal of Animal Physiology and Animal Nutrition, v.88, n.11-12, p.412-418, 2004b.

WASSERMAN, R.H. In: SWENSON, M.J. (Ed.). Duke's physiology of domestic animals. Bones. 10. ed. Ithaca, Cornell University Press, 1984. p. 467-485.

WHITLOCK, R.H.; SCHRYVER, H.F.; KROOK, L. et al. The effects of high dietary calcium for horses. In: American Association of Equine, 16 th. Anais..., 1970. p.127

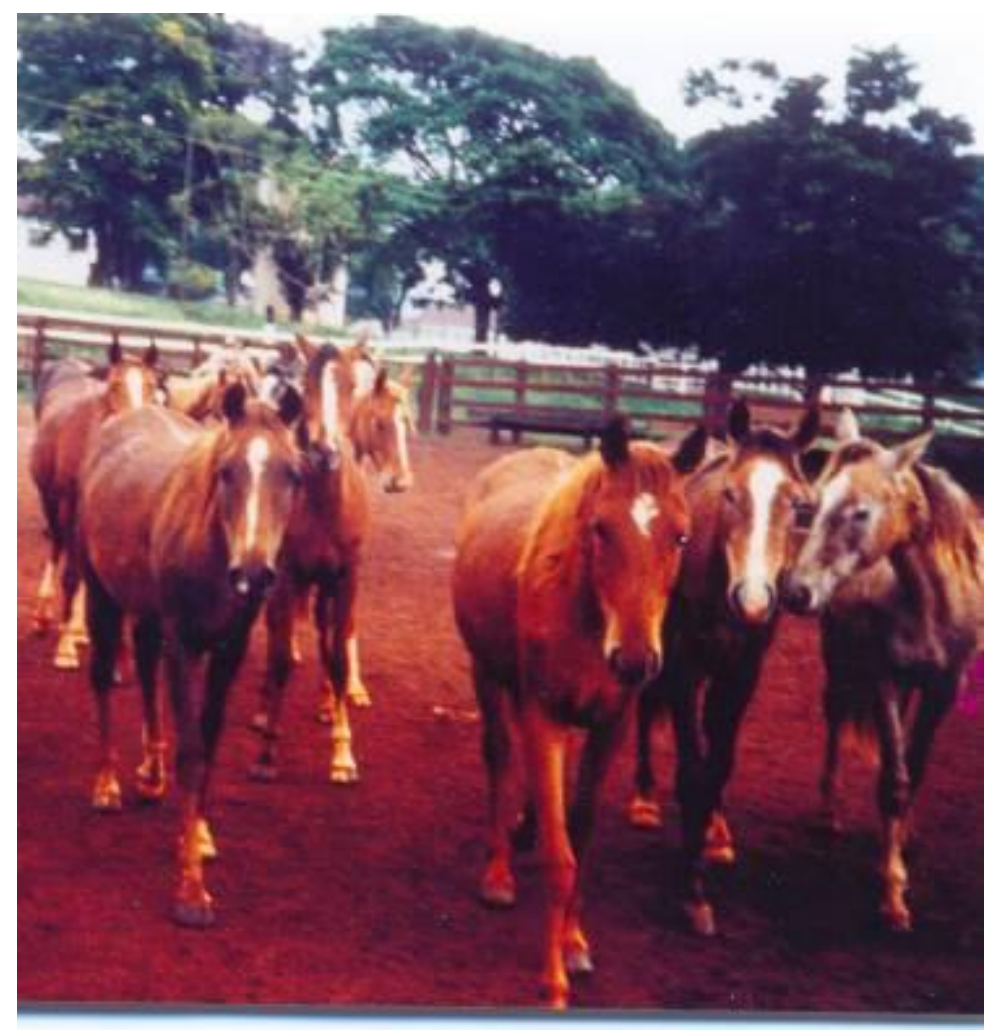

Figura 3 - Potras mestiço árabe e árabe utilizadas no experimento 